\title{
ESTUdO NUMÉRICO AERODINÂMICO DA APLICAÇÃO DE GURNEY FLAP EM UMA ASA TRASEIRA DE UM CARRO DE CORRIDA
}

\author{
Bruno Ricardo Massucatto Padilha ${ }^{1}$ e Gustavo Felipe Plattau ${ }^{2}$ \\ ${ }^{1}$ Instituto Tecnológico da Aeronáutica - ITA \\ ${ }^{2}$ Universidade Tecnológica Federal do Paraná - UTFPR \\ E-mails: bruno.massucatto@gmail.com, plattau@hotmail.com
}

\section{RESUMO}

Gurney Flaps são geometrias de controle de fluxo passivas, aplicadas em superfícies de sustentação, posicionadas no bordo de fuga e sobre a face de alta pressão da superfície. Estes dispositivos promovem a geração de vórtices, que acrescentam diferença de pressão entre as superfícies superiores e inferiores de uma asa qualquer e, consequentemente, geram maior sustentação. Sua simples geometria permite uma ampla gama de utilização, tanto no âmbito aeronáutico quanto Motorsport. Curiosamente, algumas categorias de Turismo não fazem uso de Gurney Flap em seus veículos. Para demonstrar os resultados da aplicação de tal geometria, propõe-se o estudo numérico de uma asa hipotética, dividido em três etapas: estudo do comportamento bidimensional do perfil hiper-sustentador; modelagem tridimensional da asa por meio da ferramenta Autodesk Inventor; e por fim, simulação do escoamento sobre a asa utilizando o software Autodesk CFD, esta subdivida em 2 fases: asa na condição de máximo ângulo de ataque, sem a aplicação do Gurney Flap e na sequência, com mesmo ângulo de ataque, utilizando diversas configurações de tamanhos de Gurney Flap, variando-os em razão da corda, na ordem de 1 a $4 \%$ do valor da mesma. Como resultado, avalia-se a sustentação, arrasto e os coeficientes de sustentação e arrasto, além da relação $C_{L} / C_{D}$, no intuito de identificar a asa mais eficiente.

\section{INTRODUÇÃO}

Geometrias de controle de fluxo passivas baseiam-se no princípio de energização da camada limite por linhas de vórtices convectadas a partir das protuberâncias [8]. Esta energização resulta em uma maior dificuldade de descolamento da camada limite, promovendo maior eficiência em altos ângulos de ataque. Esta caraterística é muito apreciada para aplicações em asas fixas, o que pode significar maior controlabilidade por meio de maior eficiência do escoamento por sobre superfícies de comando, bem como asas rotativas, que por sua vez, pode apresentar melhorias em condições próximas ao estol ou estol dinâmico das asas. Para números de Reynolds mais altos, onde as caraterísticas do escoamento tornam-se turbulento [4], as geometrias de controle passivo de escoamento podem apresentar fatores determinantes para a eficiência da asa.

No campo da engenharia aeronáutica, consideráveis esforços são aplicados para a melhora do desempenho aerodinâmico de um aerofólio, tais como aumento de sustentação, redução de arrasto, entre outros parâmetros. Diversas outras áreas beneficiam-se com os avanços da 
engenharia, como exemplo de tecnologia ao estado da arte, carros de competição de alto desempenho apresentam inúmeras tecnologias originalmente desenvolvidas para aplicação aeronáutica.

A aerodinâmica tem impacto significante no desempenho do carro de corrida moderno. Um veículo com uma carroceria com design eficientemente aerodinâmico, apresenta considerável redução de arrasto, fazendo melhor uso da potência do motor, permitindo elevadas velocidades nas retas. Em contrapartida, altas velocidades impõem restrições à manobrabilidade do veículo, ultrapassando com frequência a capacidade de aderência do mesmo. Uma das soluções para tal problema é o uso de asas, que por sua vez, são capazes de gerar pressão aerodinâmica, aumentando a aderência com o solo. Mais uma vez, a aerodinâmica desempenha um papel essencial à performance do veículo.

A downforce é um componente aerodinâmico que exerce uma força normal ao veículo, isto é, perpendicular à velocidade do mesmo. Esta força aumenta as forças de reação normal agindo sobre os pneus, que por sua vez, aumentam o atrito estático máximo que os pneus são capazes de sustentar, aumentando significativamente os limites de aderência. Isso se traduz em velocidades angulares mais elevadas. No entanto, a geração de downforce também produz arrasto induzido, o que penaliza o desempenho em altas velocidades. Estes efeitos indesejáveis podem ser contornados por soluções de engenharia, de modo que o trade-off pode ser alcançado. Como exemplo, o uso de dispositivos moveis, como o Drag Reduction System (DRS) na Fórmula 1, e uso de flaps em aeronaves, são exemplos claros de dispositivos usados para modificação do desempenho aerodinâmico das asas. Outra maneira encontrada para redução de arrasto é a introdução de geometrias locais para modificar o fluxo de ar, ou seja, geometrias de controle de fluxo.

Com a disponibilidade de ferramentas de projeto, ensaios e análise aerodinâmicas cada vez mais sofisticadas, os projetos de veículos de competição tornaram-se cada vez mais complexos e eficientes. Buscando ganho de performance em cada parte do veículo, diversas soluções baseadas em geometrias de controle de fluxo passivas foram adotadas, e continuam sendo usadas até os dias atuais, como podemos observar na Figura 1.

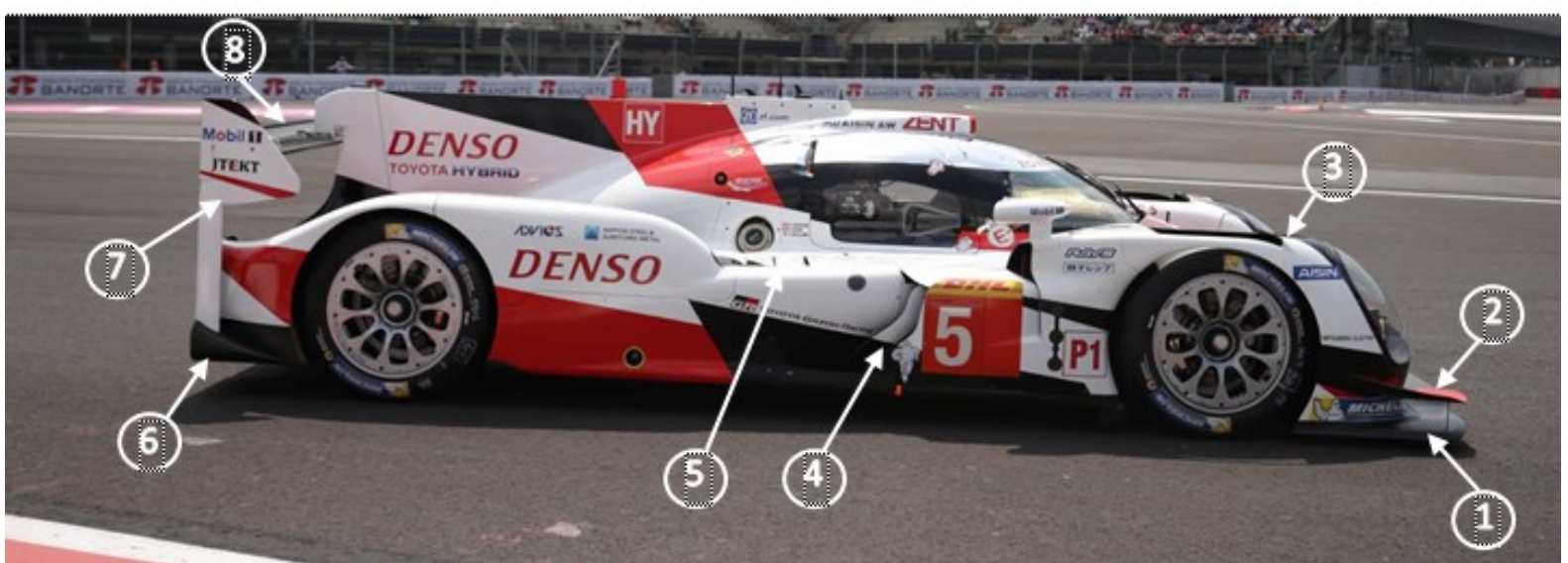

(1) Splitter (2) Canards (3) Defletor (4) Side duct (5) Flip-Ups (6) Difusor (7) Endplate (8) Gurney Flap

Figura 1 - Exemplo de aplicações de geometrias de controle de fluxo passiva em um veículo de corrida categoria FIA- World Endurance Championship. Fonte: http://www.toyotagazooracing.com. 
Uma destas soluções, foco deste trabalho, é o Gurney Flap, um dispositivo simples e eficiente, capaz de transformar qualquer aerofólio numa complexa peça aerodinâmica. Encontrado nos projetos mais modernos de carros de corrida, o Gurney Flap é uma pequena aleta instalada no bordo de fuga da asa. Esta aleta cria pequenos vórtices que intensificam a diminuição da espessura da camada limite no lado inferior, provocando o aumento de pressão no lado superior e diminuição no lado inferior, e consequentemente, gerando maior sustentação.

Tendo em vista sua ampla utilização, a proposta deste trabalho é analisar numericamente os efeitos do Gurney Flap em uma asa traseira de um veículo de turismo, através da simulação do escoamento de ar sobre a asa, e assim avaliar os coeficientes de arrasto e de sustentação, além da relação $\mathrm{C}_{\mathrm{L}} / \mathrm{C}_{\mathrm{D}}$, no intuito de identificar a asa mais eficiente, além de fornecer/contribuir com informações iniciais para equipes de corrida, Fórmula SAE, entre outras categorias de interesse.

\section{CONTEXTO HISTÓRICO}

O Gurney Flap é um dispositivo de controle de fluxo passivo que leva o nome do seu criador, Dan Gurney. Gurney chegou ao flap em 1971, quando ele e Bobby Unser testavam um carro novo no Phoenix International Raceway, e Unser se queixou do desempenho do carro em curvas. Gurney lembrou então de alguns experimentos feitos nos anos 50, que utilizavam spoilers para minimizar a sustentação aerodinâmica. $\mathrm{Na}$ época, os spoilers ainda não eram vistos como um elemento que pudesse maximizar a tração pelo aumento da carga vertical, resultando em maior aderência, porém eles queriam apenas mitigar a sustentação aerodinâmica gerada pela própria carroceria do carro e interação com o solo [2].

Gurney imaginou que se fosse instalado um spoiler na asa traseira, ele talvez conseguisse mitigar a sustentação da carroceria, e ao mesmo tempo, exercer determinado peso (downforce) sobre o eixo traseiro, obtendo maior tração. Em determinado momento dos testes, sem que se houvesse obter resultados satisfatórios, Dan Gurney acrescentou uma lâmina de alumínio a asa traseiro do veículo, instalando-a na extremidade traseira da mesma, no sentido vertical, e Unser retornou a pista para novos testes. Como resultado, os tempos não melhoraram, mas Unser explicou que isso ocorreu porque a traseira tinha tanta downforce que o carro estava saindo de frente por estar com a dianteira muito leve. Os mesmos ajustes foram então feitos na asa dianteira e o carro voltou a ter equilíbrio [2].

Unser e Gurney tentaram manter a descoberta em segredo, afirmando que o spoiler era, na verdade, um dispositivo de segurança para não machucar as mãos quando o carro estivesse sendo empurrado. Por incrível que pareça, a história foi convincente e Gurney conseguiu usar seu flap por várias temporadas antes que as outras equipes descobrissem sua real função e copiassem em seus carros [4].

Após esses acontecimentos, Gurney reuniu-se com o designer aerodinamicista Bob Liebeck, da Douglas Aircraft Company, a quem explicou seus princípios. Liebeck testou os flaps e comprovou sua eficiência. Eles ganharam o nome "Gurney Flaps", e a Douglas Aircraft Company os adotou em seus projetos aeronáuticos. Atualmente, os Gurney Flaps são usados em spoilers de carros de rua, asas de carros de corrida, estabilizadores de helicópteros e asas de aviões [2]. 
Os resultados a seguir foram tão satisfatórios que as demais equipes passaram a utilizar tais recursos, sem ao menos compreender os fenômenos aerodinâmicos envolvidos e até hoje, décadas após os primeiros experimentos, o Gurney Flap se faz presentes nos veículos de competição moderno, como pode-se observar na Figura 2.

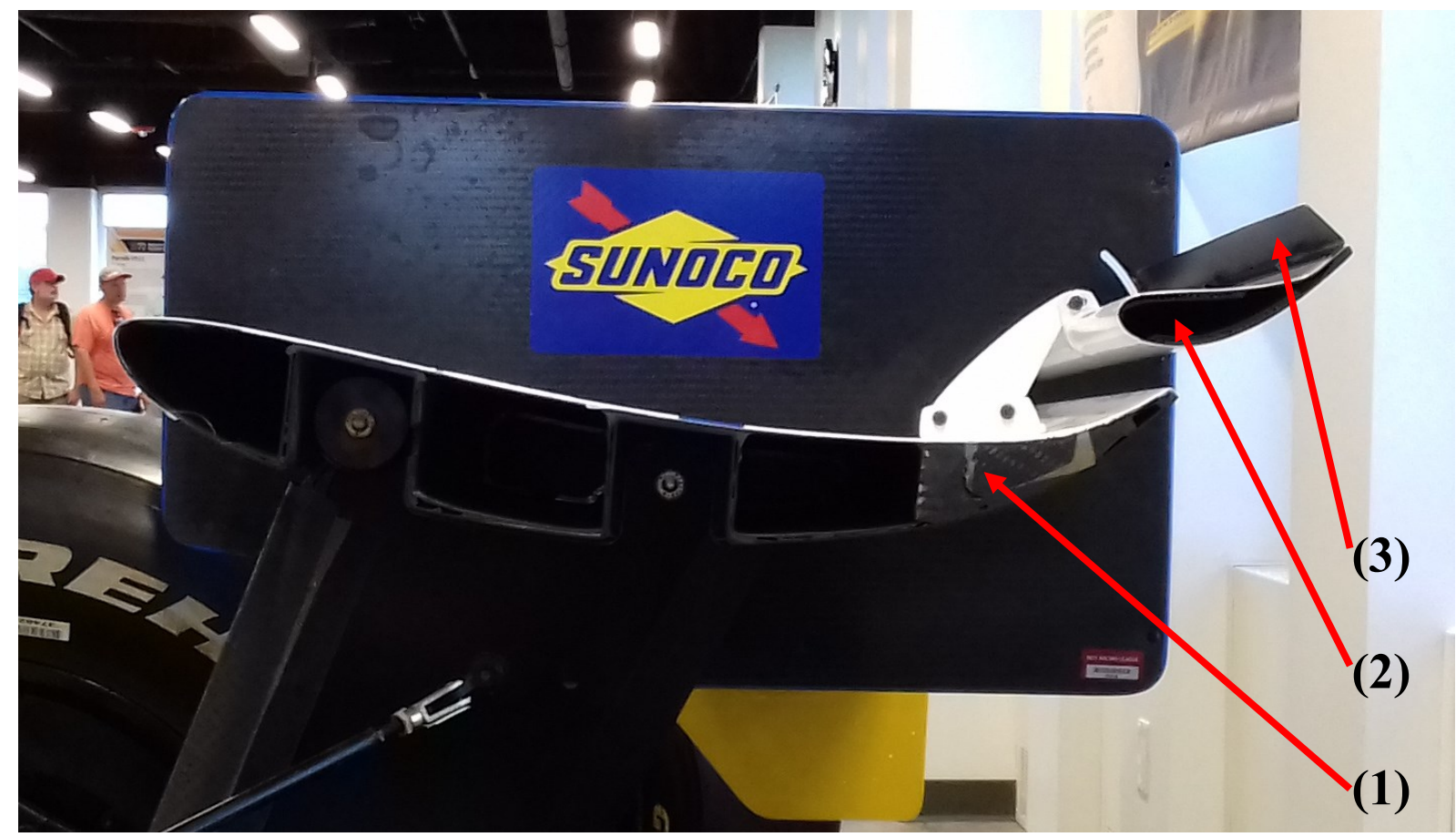

Figura 2 - Exemplo de conjunto de asa traseira de um Fórmula Indy moderno, onde observa-se a asa principal (1) e a asa secundária (2), dotada de Gurney Flap (3). Fonte: O Autor

\section{PRINCÍPIO AERODINÂMICO}

O controle da camada limite é um desafio para se obter a máxima performance em qualquer tipo de aerofólio. Em veículos de competição, onde normalmente os aerofólios encontram-se em altos ângulos de ataque, associado à turbulência envolvida no escoamento sobre o veículo, o desafio torna-se ainda maior.

O Gurney Flap atua como uma espécie de gerador de vórtices, energizando a camada limite. O efeito físico da presença do Gurney Flap é a alteração da condição de Kutta no bordo de fuga do aerofólio. Essa condição prescreve que em um aerofólio fino ocorre a geração de vórtice no sentido da face de alta pressão do aerofólio para a face de baixa pressão [1].

Com o uso do Gurney Flap, a formação destes vórtices se alteram, de forma que a sucção gerada pela barreira fisica do Gurney Flap gera vortices contra rotativos, energizando a camada limite e acelerando o fuído, de modo a gerar acréscimo de coeficiente de sustentação $(C L)$, e tipicamente, o arrasto $(C D)$. Porém, a relação $C_{L} / C_{D}$ é otimizada com o dimensionamento correto do Gurney Flap em relação a porcentagem da corda da asa $(c)$ [5]. A Figura 3 apresenta esquematicamente o princípio do funcionamento aerodinâmico do Gurney Flap. 


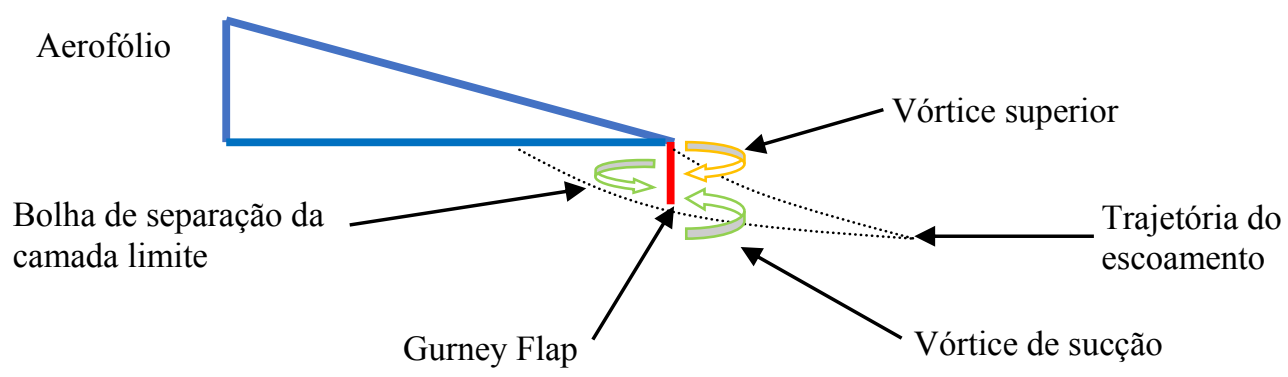

Figura 3: Princípio aerodinâmico do funcionamento do Gurney Flap. Fonte: O Autor.

\section{ESTUDO DE CASO}

Com objetivo de analisar um modelo mais próximo de um caso real possível, pesquisou-se diversas categorias do automobilismo Brasileiro, sendo que identifica-se a possibilidade de aplicação ao modelo de asa traseira da categoria Stock Car Light, onde a mesma não faz uso do Gurney Flap, como apresentado na Figura 4.

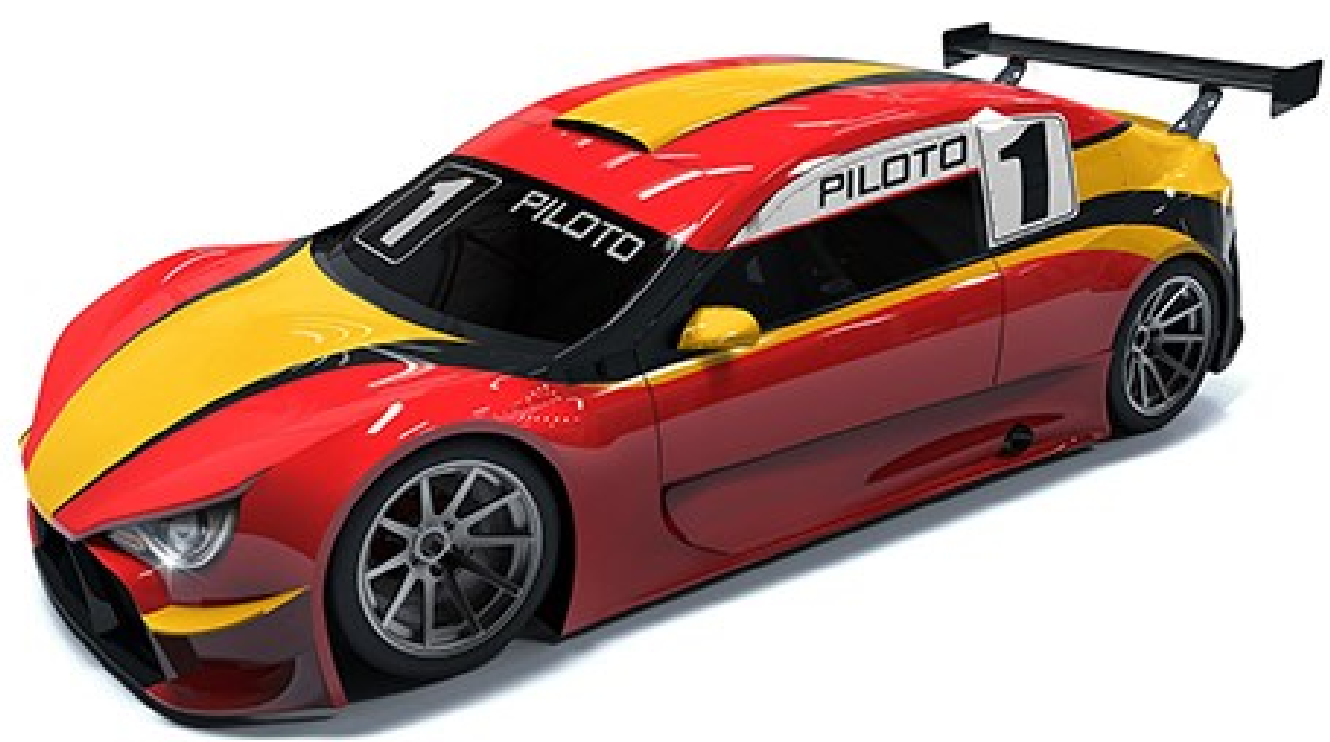

Figura 4: Modelo de veículo da categoria Stock Car Light. Fonte: http://www. jlind.com.br.

Assim, por meio de informações disponíveis no site do fabricante do carro da categoria [3], modela-se tridimensionalmente a asa, respeitando as medidas de corda $(c)$ e envergadura $(b)$, conforme a Figura 5:
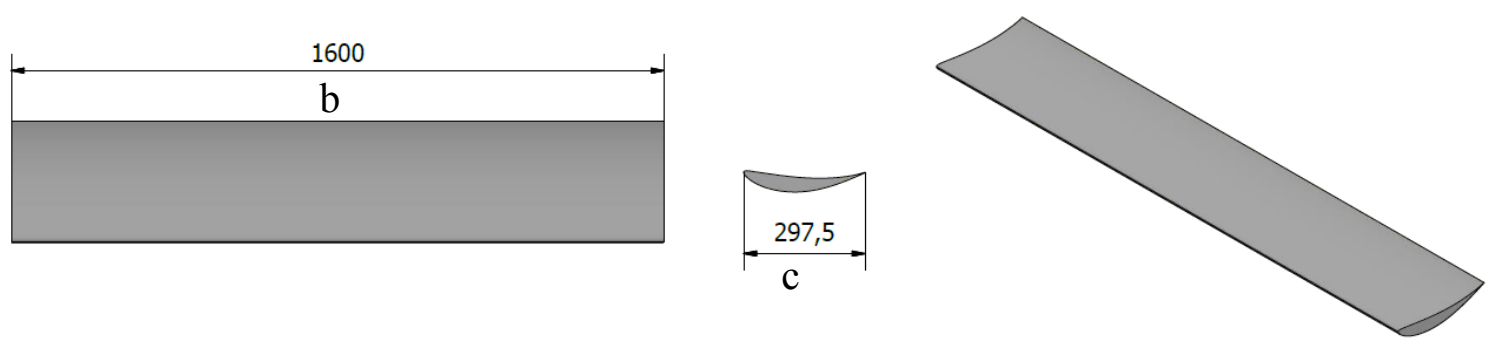

Figura 5 - Dimensões da asa projetada e ensaiada (Medidas em milímetros). Fonte: O Autor. 
Como não é possível o acesso ao perfil utilizado, por questões relacionadas a segredo de projeto, escolheu-se então o perfil $\mathrm{CH} 10$, um perfil de alta sustentação e com câmber, muito utilizado nesta aplicação e disponível no site UIUC Airfoil Data Site [9], conforme Figura 6.

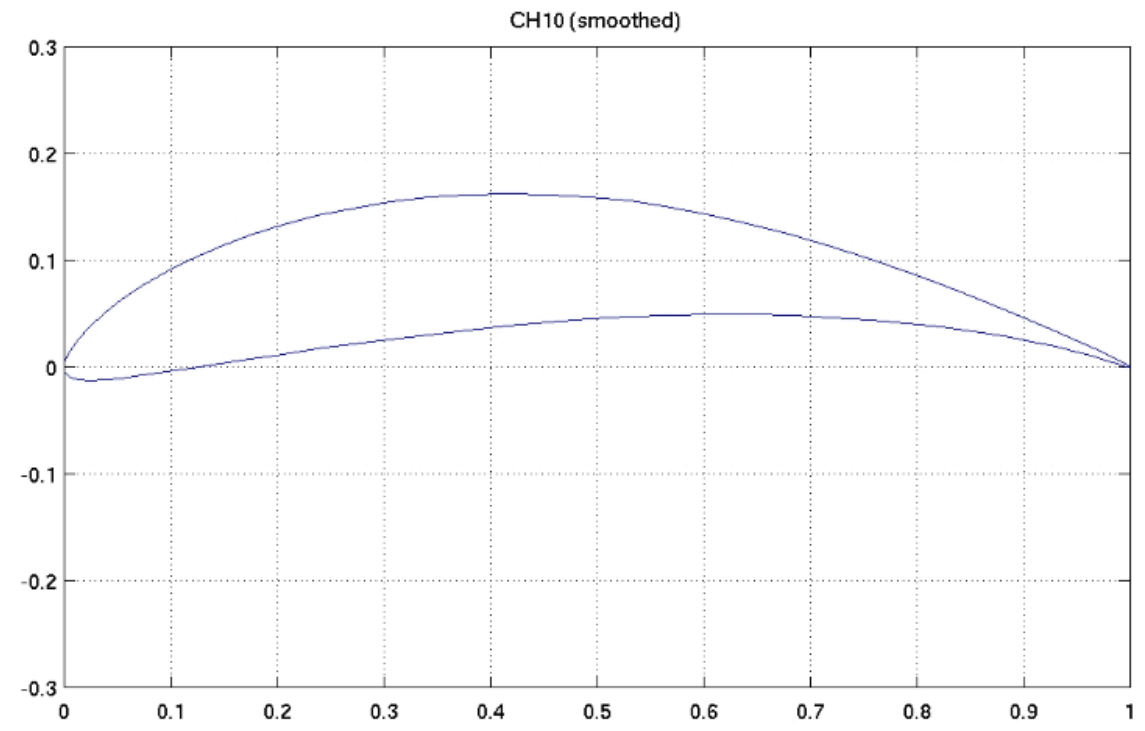

Figura 6 - Perfil CH10. Fonte:http://m-selig.ae.illinois. edu/ads/coord_database.html

Para a geração da simulação computacional, o software utilizado foi o Autodesk CFD. A escolha foi justificada pelo motivo que a empresa disponibiliza licenças acadêmicas sem restrições de performance, como por exemplo, número de elementos da simulação. Assim, os parâmetros da simulação foram os mesmos sugeridos por [6], o qual em trabalho semelhante de validações aerodinâmicas por meio de ensaios em túnel de vento, obtiveram resultados satisfatórios.

Como parâmetros em comum às simulações, destacam-se:

- Número total de elementos (Abaixo de 3 milhões);

- Malha não estruturada do tipo triangular, e com ajustes sugeridos por [8] para o controle da espessura da camada da malha (Layer Factor) e progressão do tamanho dos elementos da camada (Layer Graduation), a fim de se obter uma melhor discretização da camada proximo a asa (menosres elementos) e uma discretização mais grosseira aos extremos do volume de controle (maiores elementos);

- Setup do fluído conforme atmosfera padrão (ISA): Pressão de 93640 Pa e Temperatura de $27^{\circ} \mathrm{C}$;

- Material da asa definido como plástico tipo ABS (Acrilonitrila Butadieno Estireno);

- Modelo de turbulência SST k-omega.

Com relação ao volume de controle, foram levadas em consideração duas variáveis:

- $\mathrm{O}$ fato de que a asa real utiliza end plate: $\mathrm{O}$ end plate é uma estrutura física localizada nas pontas da asa que tem como função eliminar os vórtices de ponta de asa e assim 
reduzir o arrasto induzido. Levando em consideração que o regulamento da categoria não fornece as medidas do mesmo e o presente trabalho não visa um estudo de eficiência do end plate ou sua interferência no Gurney Flap, as paredes do volume de controle são coincidentes com as pontas da asa, eliminado assim possíveis vórtices de ponta de asa.

- Levando em consideração os resultados obtidos por [6] e a possibilidade de utilização do túnel de vento disponível no ITA - Instituto Tecnológico da Aeronáutica para as simulações, o volume de controle apresenta um volume conpatível com o volume de umas seção típica de testes de um túnel transônico.

Novamente, seguindo as recomendações de [6], foi desenvolvida uma malha com camadas de prisma sobre o modelo simulado a fim de se obter uma melhor captura dos fenômenos da camada limite. Assim, para todas as simulações foram desenvolvidas malhas com 15 camadas de prismas sobre a asa. Essa condição exige que a solução dos termos de advecção do modelo simulado seja o Petrov-Galerkin modificado. O resultado pode ser observado na Figura 7:

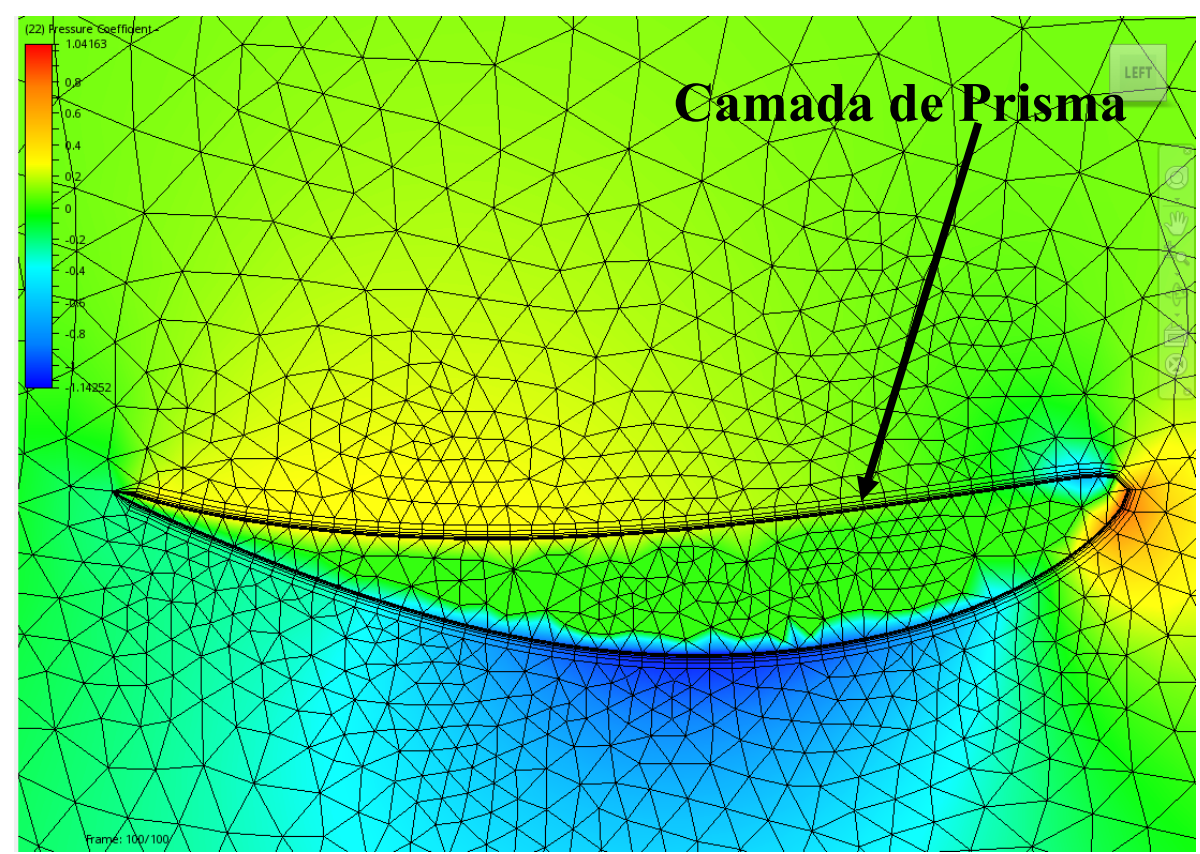

Figura 7 - Camada de prisma sobre o modelo de asa utilizado para a simulação. Fonte: $\mathrm{O}$ autor.

A Tabela 1 apresenta as características em comum dos ensaios (velocidade e ângulo de ataque), bem como o número aproximado de elementos da malha gerada para cada simulação.

Tabela 1 - Padrões utilizados nas simulações. Fonte: $\mathrm{O}$ autor.

\begin{tabular}{|c|c|c|c|}
\hline Tipo de asa & $\begin{array}{c}\text { Ângulo de } \\
\text { ataque } \boldsymbol{\alpha}\left[{ }^{\circ} \mathbf{]}\right.\end{array}$ & $\begin{array}{c}\text { Velocidade } \\
{[\mathbf{m} / \mathbf{s}]}\end{array}$ & $\begin{array}{c}\text { Número aproximado de } \\
\text { elementos }\end{array}$ \\
\hline Asa sem Gurney Flap & 10 & 70 & 2.893 .066 \\
\hline Asa com Gurney Flap 1\% c & 10 & 70 & 2.943 .789 \\
\hline Asa com Gurney Flap 2\% c & 10 & 70 & 2.954 .654 \\
\hline Asa com Gurney Flap 3\% c & 10 & 70 & 2.879 .948 \\
\hline Asa com Gurney Flap 4\% c & 10 & 70 & 2.967 .938 \\
\hline
\end{tabular}

Legenda: $\mathrm{c}=$ corda 
A convergência da simulação é obtida de forma automática por meio da minimização do resíduo da simulação numérica. Para as simulações determinadas pela matriz de ensaio, foi estabelecido o valor máximo de 5000 iterações, onde todas as convergências foram obtidas na ordem aproximada de 800 a 1300 iterações. Durante o processamento é possível observar graficamente a evolução das iterações numéricas por meio gráfico, como apresentado na Figura 8.

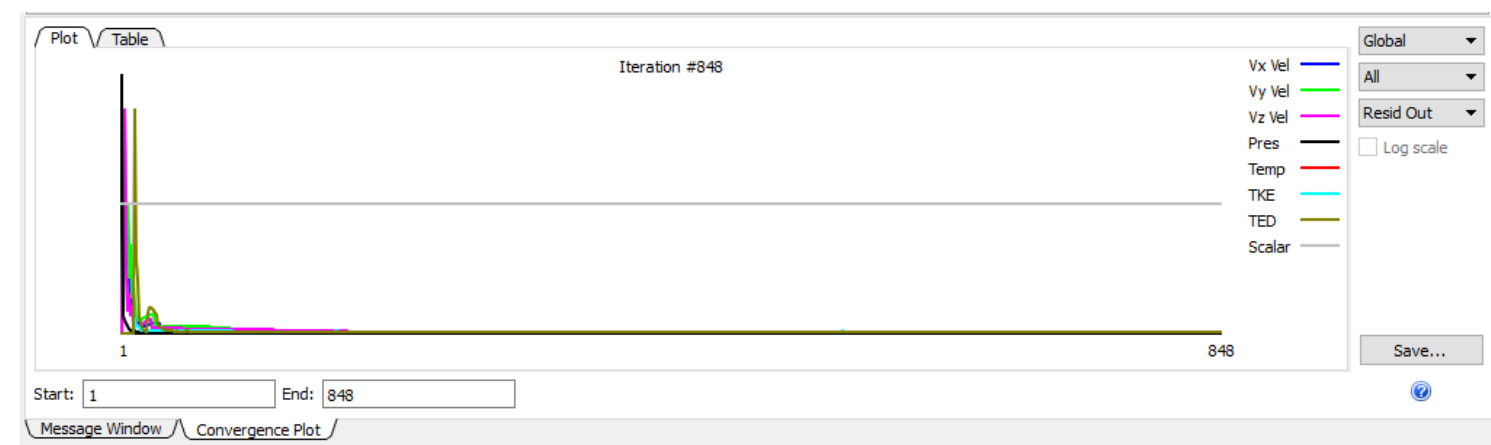

Figura 8 - Representação gráfica do software Autodesk CFD, o qual é possivel observar a progressão dos valores residuais oriundos das iterações até a convergência. $\mathrm{O}$ autor.

\section{RESULTADOS E DISCUSSÕES}

Os resultados obtidos pelas simulações podem ser analisados de modo qualitativo e quantitativo. Do ponto de vista quantitativo, é possível observar o comportamento do escoamento sobre a asa analisada, e observar particularidades relacionadas a formação da esteira bidimensional do escoamento, bem como as alterações do seu comportamento devido a alteração da geometria da asa.

Na condição das simulações desenvolvidas, é possível observar claramente os efeitos da alteração geométrica da asa, ou seja, o aumento do Gurney Flap, em relação ao comportamento bidimensional da esteira, onde percebe-se as alterações relacionadas a velocidade do escoamento e a formação da esteira após o perfil, como observada na Figura 9. 
1. Gurney Flap 1\%; Velocidade $70 \mathrm{~m} / \mathrm{s}$; Alpha 10\%.

2. Gurney Flap 2\%; Velocidade $70 \mathrm{~m} / \mathrm{s}$; Alpha 10\%.

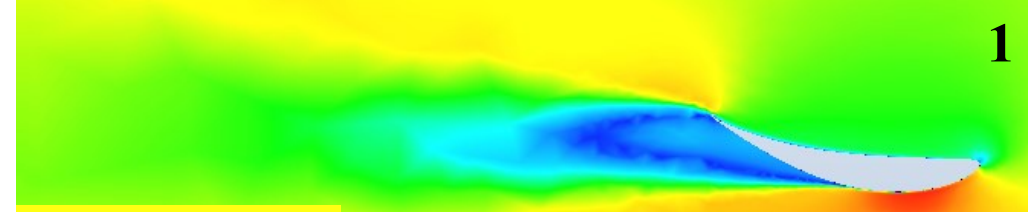

(1) Velocity Magnitude - $\mathrm{m} / \mathrm{s}$

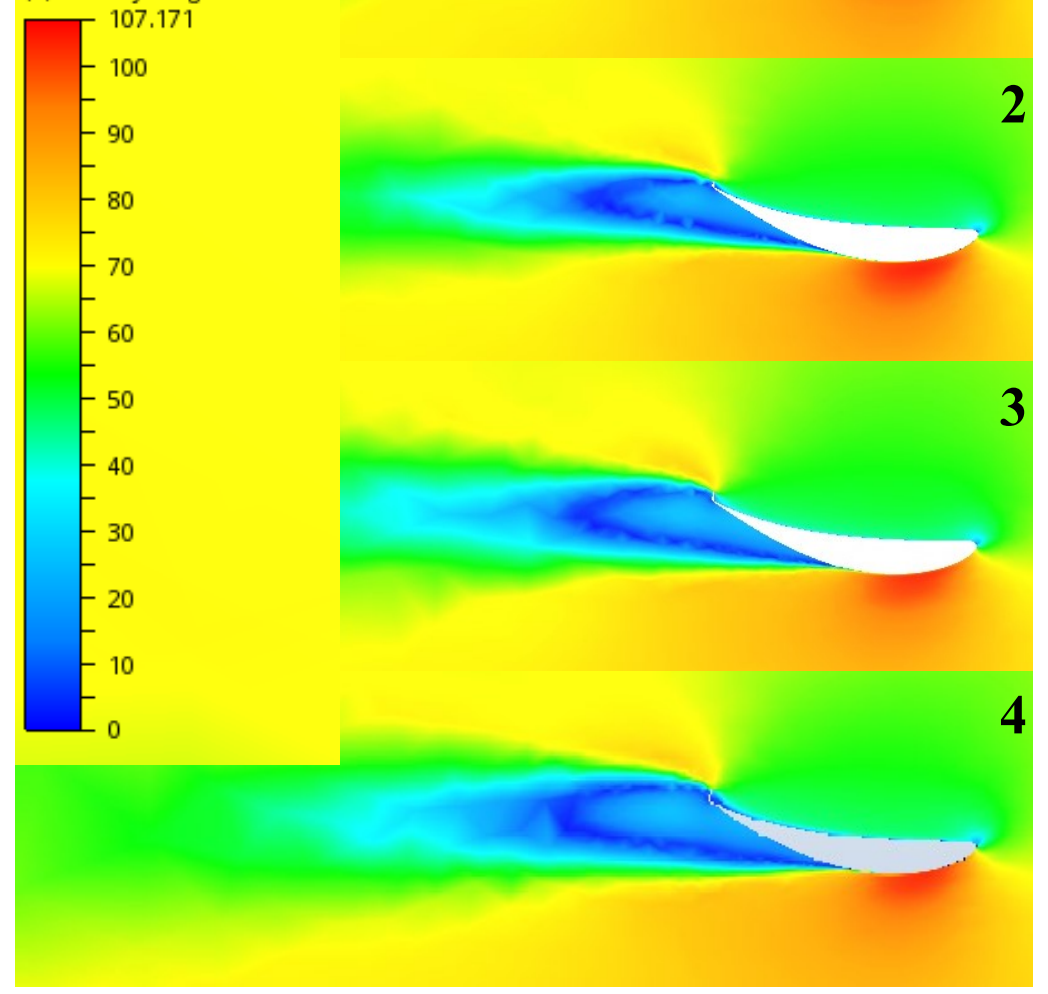

4. Gurney Flap 4\%; Velocidade $70 \mathrm{~m} / \mathrm{s}$; Alpha 10\%.

3. Gurney Flap 3\%; Velocidade $70 \mathrm{~m} / \mathrm{s}$; Alpha 10 .

Figura 9 - Análise dos resultados qualitativos das simulações. Fonte: O autor.

A análise quantitativa dos resultados é gerada segundo [7], onde os fatores básicos principais a serem observados em um projeto de uma asa são os valores obtidos em relação a sustentação, arrasto e momento aerodinâmico. Este último fator, o momento aerodinâmico, para o projeto de uma asa para aplicação veicular não será fator determinante ou se quer cause qualquer interferência pelo fato de que a asa esteja fixa ao carro e não obtenha qualquer grau de liberdade sobre o mesmo.

Desta forma, apresenta-se de forma gráfica com intuito de promover a melhor compreensão dos dados obtidos, a comparação entre a downforce (valores em Newtons) promovida por cada asa em razão do tamanho do respectivo Gurney Flap (em \% de corda da asa), como apresentado na Figura 10. 


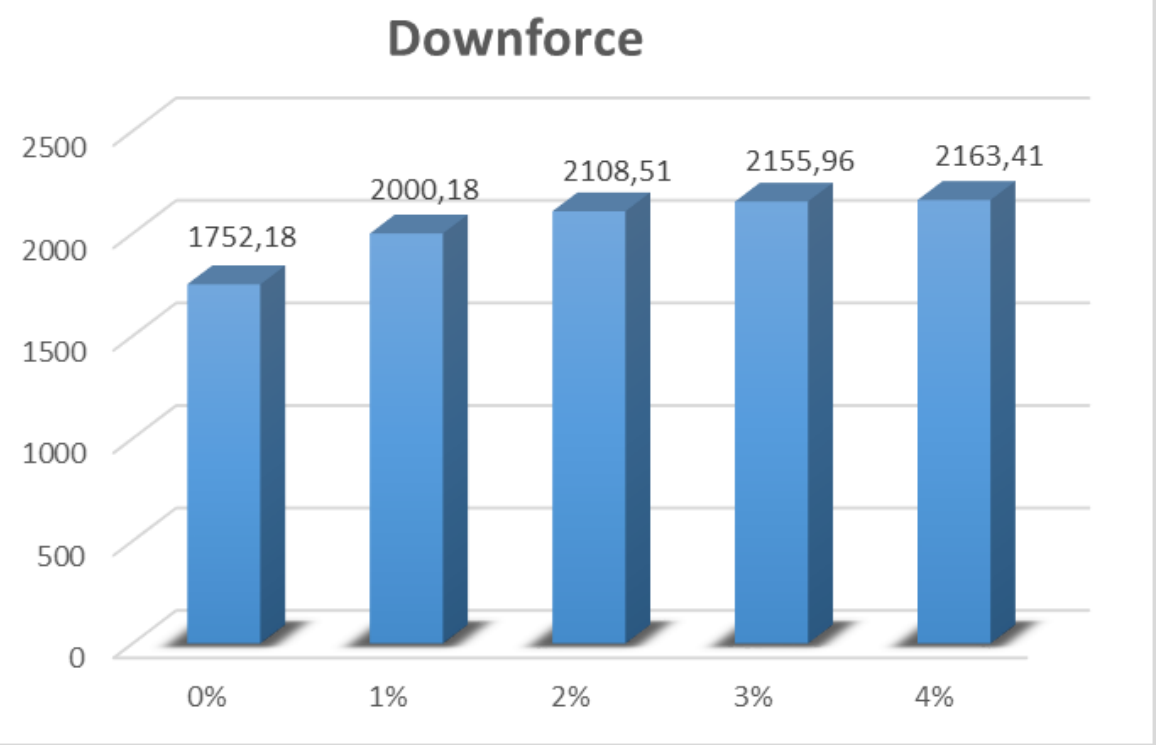

Figura 10 - Relação entre a Downforce valores em Newtons (N) obtido pelas asas simuladas em relação ao tamanho do Gurney Flap (\% da corda da asa).

Na sequência, avalia-se o impacto da adição do Gurney Flap, bem como da variação do seu tamanho no arrasto total gerado pelas asas simuladas, todas dentro das mesmas condições de atmosfera, velocidade e ângulo de ataque, onde a Figura 11 apresenta tais resultados na relação da força de arrasto gerada em Newtons e o tamanho do Gurney Flap em porcentagem de corda da asa.

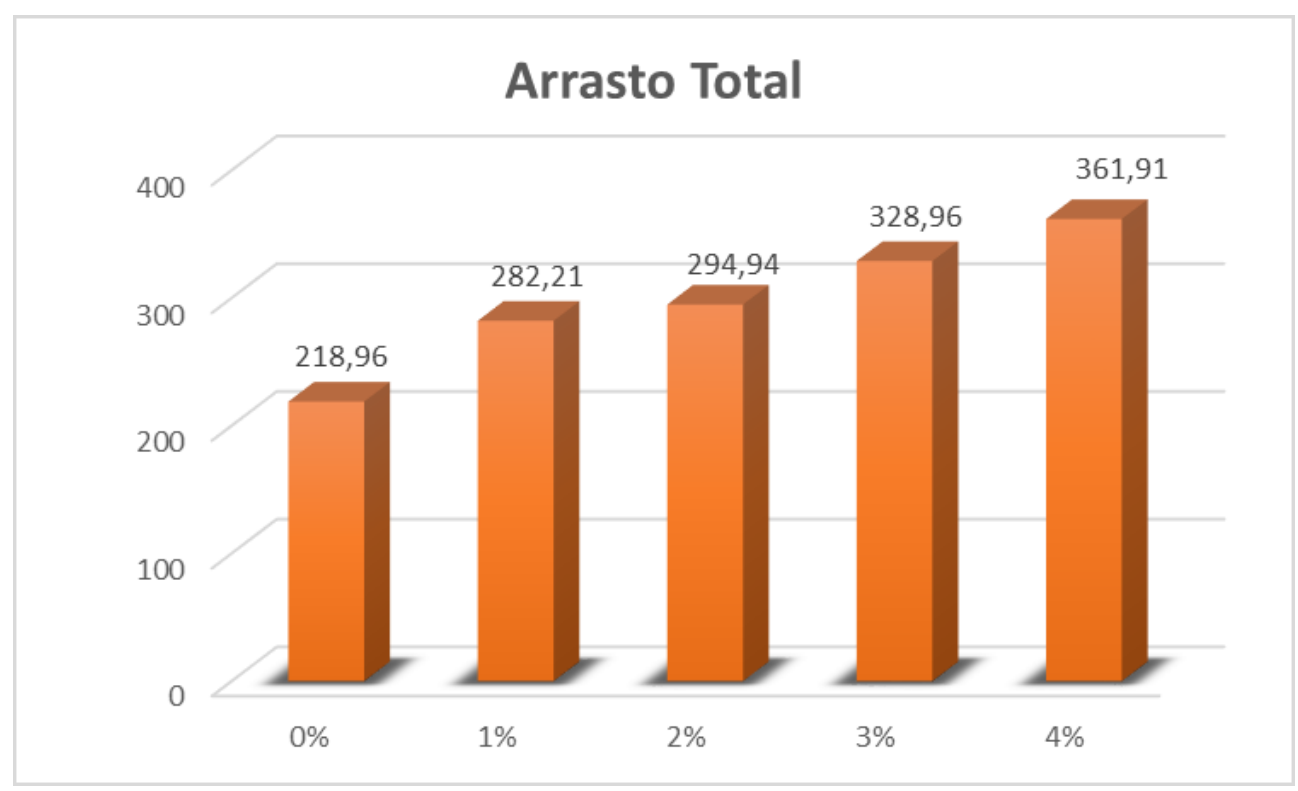

Figura 11 - Relação entre o Arrasto Total da asa (valores em Newtons) obtido pelas asas simuladas em relação ao tamanho do Gurney Flap (\% da corda da asa).

É interessante observar que os valores de sustentação e arrasto aumentam, sem uma proporção clara, em razão da alteração do tamanho do Gurney Flap em porcentagem da corda. Desta forma, novamente citando [7], se faz necessário identificar um parâmetro de comparação entre os valores (downforce e arrasto) que possa indicar um valor de Gurney Flap em 
porcentagem de corda mais eficiente, ou seja, que proporcione aumento de downforce gerada sem demasiada punição pela geração do arrasto aerodinâmico total da asa.

Para tal, [7] sugere que a relação entre o coeficiente de sustentação $\left(C_{L}\right)$ e o coeficiente de arrasto $\left(C_{D}\right)$, ou seja, o maior valor obtido pela relação $C_{L} / C_{D}$, possa indicar a asa mais eficiente, que em outras palavras, promova o aumento da sustentação com a menor geração de arrasto proporcionalmente.

E, de acordo com [1], é possível através da Equações 1, Downforce $(L)$ e da Equação 2, Arrasto Total $(D)$, obter os valores de $C_{L}$ e $C_{D}$ das respectivas asas.

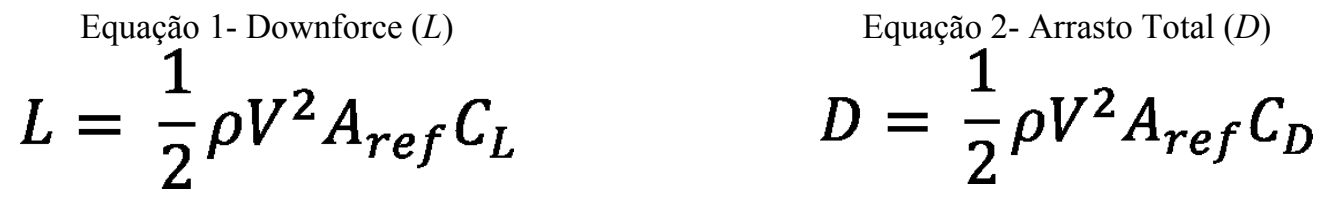

Uma vez obtidos tais coeficientes, é possível determinar a relação $C_{L} / C_{D}$ e obter assim o tamanho de Gurney Flap em porcentagem da corda que apresenta a melhor performance aerodinâmica, e desta forma, apresenta-se graficamente na Figura 12.

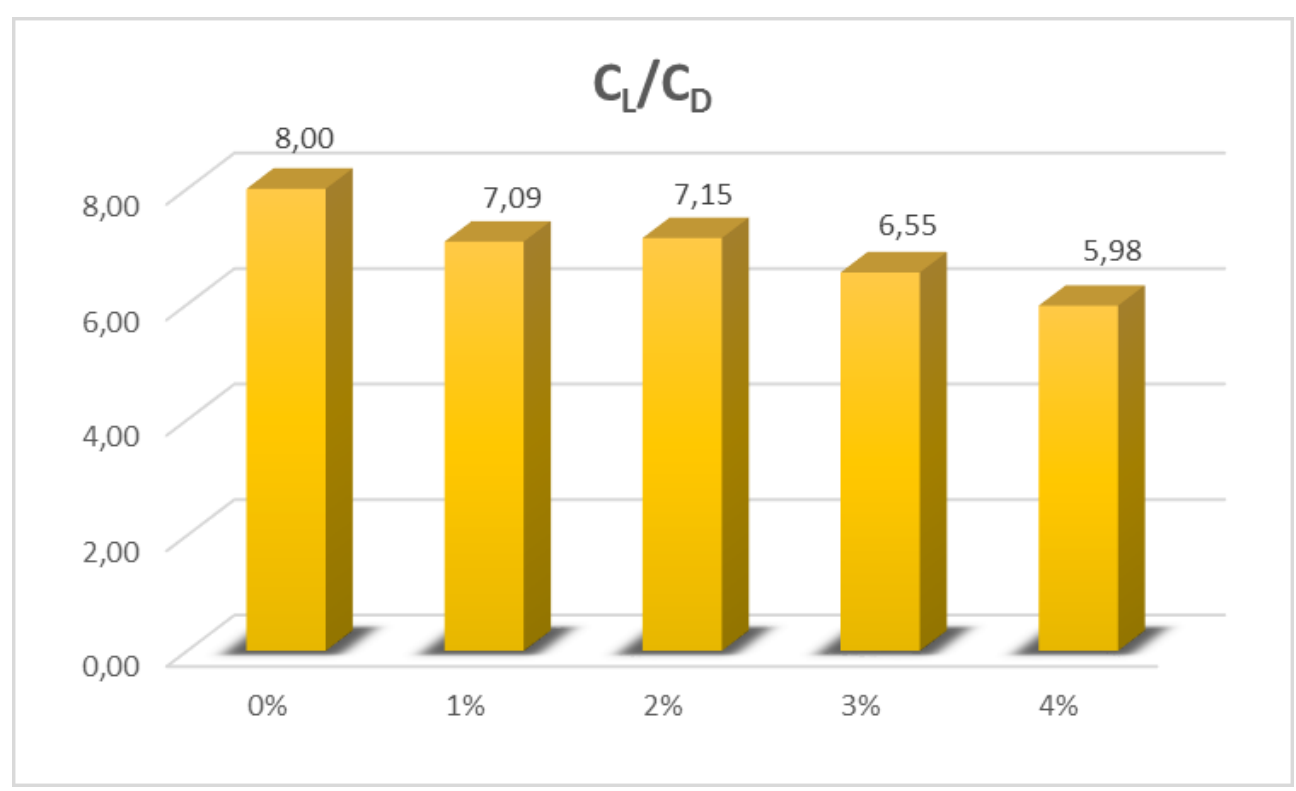

Figura 12 - Relação $C_{L} / C_{D}$ entre as asas simuladas para identificação da asa dotada de Gurney Flap mais eficiente.

O resultado gráfico da Figura 12 mostra que, em razão da eficiência aerodinâmica, a asa em a adição do Gurney Flap apresenta resultados superiores em relação as asas as quais foram submetidas a adição de tais recursos aerodinâmicos. Porém, observando-se a Figura 10 é notável a adição de sustentação por meio da adição de tal recurso.

Sendo assim, considerando-se os benefícios da adição do Gurney Flap, observa-se que dentre as simulações realizadas, a asa com melhor relação $C_{L} / C_{D}$ obtida é a asa com o Gurney Flap projetado em razão de $2 \%$ do tamanho da corda. O valor de $C_{L} / C_{D}$ superior para a asa com 
razão de tamanho de Gurney Flap de $2 \%$ de corda em relação as asas com os demais tamanhos são plausíveis com os resultados e observações sugeridos por [5] e [8] como tamanho de Gurney Flap ideal.

Ainda, como critério de conclusão em relação ao tamanho do Gurney Flap ideal, a Figura 13 tem como objetivo apresentar a queda do rendimento de tal geometria a partir do tamanho de $2 \%$ em fração da corda, onde torna-se claro que o acréscimo percentual de downforce gerado é muito inferior ao arrasto total gerado para a mesma geometria, justificando-se assim claramente a não adoção de geometrias com tamanhos de Gurney Flaps superiores a 2\% da corda do perfil.

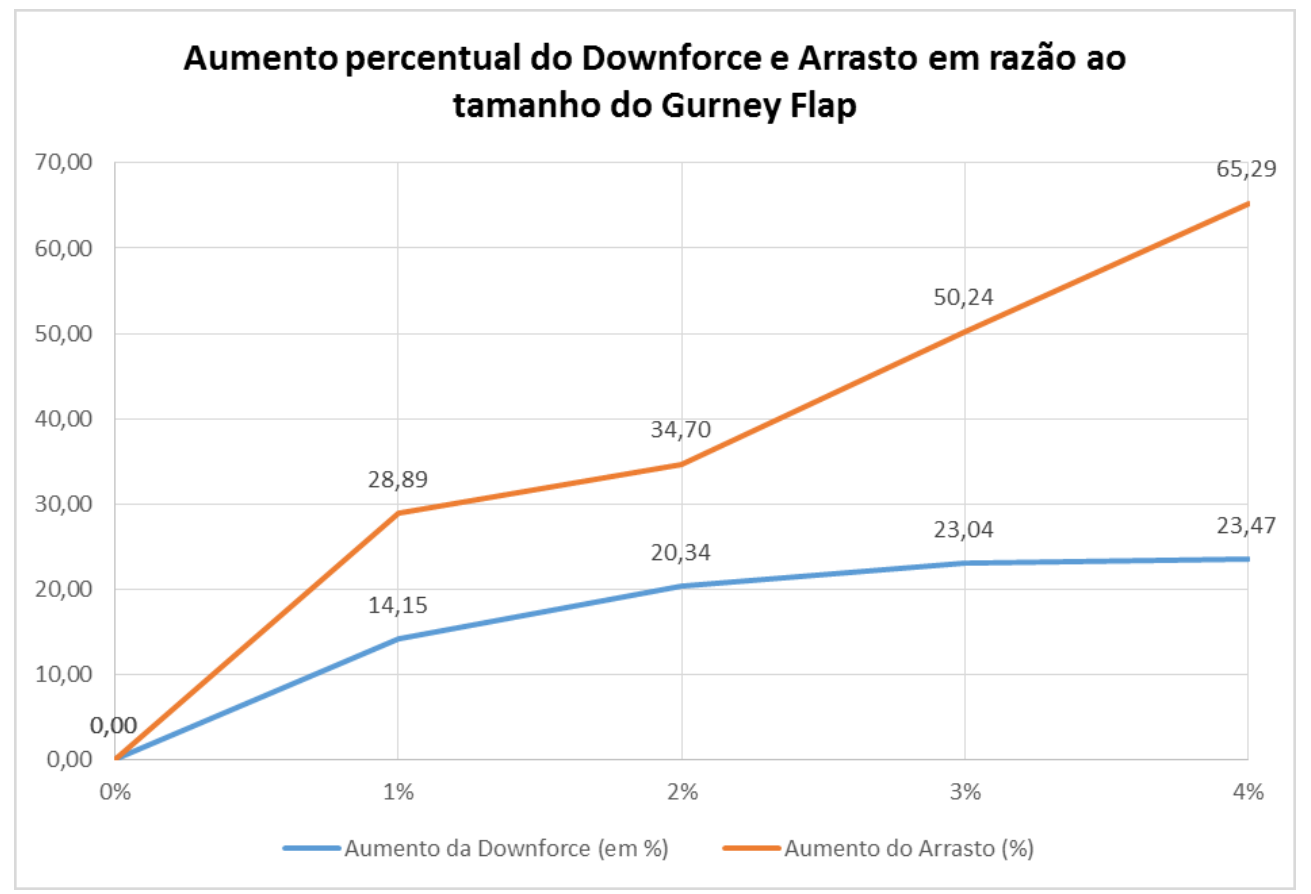

Figura 13 - Relação entre o aumento percentual do Downforce e Arrasto gerado por cada asa e respectivo tamanho de Gurney Flap.

\section{CONCLUSÃO}

Do ponto de vista numérico, os resultados foram satisfatórios, comprovando que a asa com Gurney Flap de $2 \%$ da corda é realmente a mais eficiente, compatível com o que foi citado em outras bibliografias que abordam o assunto.

O software utilizado no presente trabalho também apresentou resultados satisfatórios, em relação à facilidade na simulação deste tipo de escoamento, assim como no tempo de processamento.

Como oportunidade para trabalhos futuros, agregando valor a esta pesquisa inicial, sugere-se um ensaio experimental em túnel de vento, este disponível no ITA - Instituto Tecnológico da Aeronáutica, para validação junto ao ensaio computacional, e uma investigação numérica referente os efeitos do Gurney Flap em relação ao ângulo de estol da asa, tendo em vista que há uma energização da camada limite, pode-se haver ganhos quando o referido estol for 
atrasado e consequentemente se obter uma downforce maior em relação a uma asa sem Gurney Flap. Ainda, é notável o potencial de desenvolvimento de um perfil de forma a otimizar a utilização do Gurney Flap, ou seja, a hipótese de desenvolvimento do perfil bidimensional de forma conjugada a adição à sua geometria de um Gurney Flap.

\section{REFERÊNCIAS}

[1] BREDERODE, V. d. Aerodinâmica Incompressível: Fundamentos. Lisboa-Portugal: Press, 2014.

[2] CONTESINI, L. O herói americano: Por que você precisa conhecer o legado de Dan Gurney. Disponível em https://www.flatout.com.br/o-heroi-americano-por-que-voce-precisaconhecer-o-legado-de-dan-gurney/. Acesso em: 15/04/2018.

[3] GIAFONNE, J. P. Ficha Técnica-provisoria G-12 2017. Disponível em: www.jlind.com.br/assets/public/.../pdf/ficha-tecnica-provisoria-g-12-2017-rev-05.pdf. Acesso em: 20/04/2018.

[4] KARTHIKEYAN, N.; SUDHAKAR, S.; SURIYANARAYANAN, P. Experimental studies on the effect of leading edge tubercles on laminar separation bubble. Proceedings of the 52nd AIAA Aerospace Science Meeting, National Harbor, Maryland: AIAA Paper 2014-1279, 2014.

[5] KATZ, J. Race Car Aerodynamics. Cambridge MA: Bentley Publishers, 2006.

[6] MAZI, R., \& PADILHA, B. R. (2015). A Utilização de Ferramentas Autodesk para Projetos de Biomimética na Engenharia Aeronáutica. Autodesk University Brasil . São Paulo-2015.

[7] ROSKAM. JAN, Airplane aerodynamics and performance, DARcorporation, University of Kansas, 1997.

[8] ROSTAMZADEH, N.; HANSEN, K. L; KELSO, R. M.; Dally, B. B. The formation mechanism and impact of streamwise vortices on NACA 0021 airfoil's performance with undulating leading edge modification. Physics of fluids, v. 26, n. 107101, 2014.

[9] UIUC Airfoil Data Site - UIUC Applied Aerodynamics Group. Chuch Hollinger CH 1048-13 high lift low Reynolds number airfoil. Disponível em: http://m-selig.ae.illinois. edu/ads/coord database.html. Acesso em: 03/05/2018. 